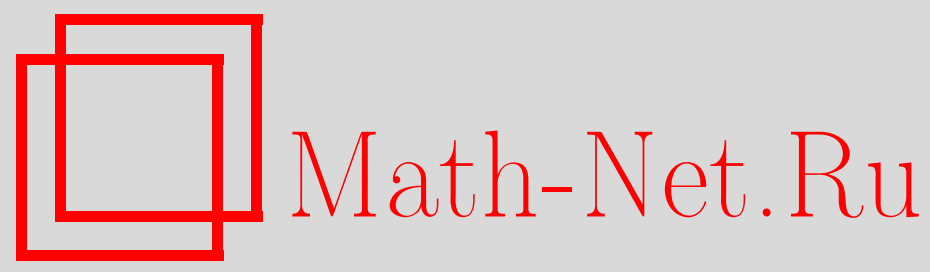

И. И. Шарапудинов, Смешанные ряды по полиномам Чебышева, ортогональным на равномерной сетке, Матем. заметки, 2005, том 78, выпуск 3, 442-465

DOI: https://doi.org/10.4213/mzm2599

Использование Общероссийского математического портала Math-Net.Ru подразумевает, что вы прочитали и согласны с пользовательским соглашением http://www . mathnet.ru/rus/agreement

Параметры загрузки:

IP: 54.224 .187 .69

26 апреля 2023 г., $17: 29: 42$

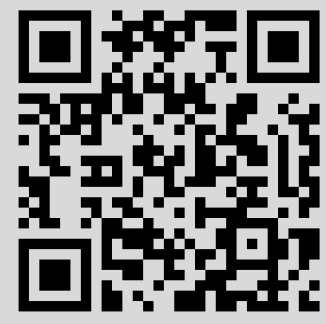




\section{СМЕШАННЫЕ РЯДЫ ПО ПОЛИНОМАМ ЧЕБЫШЕВА, ОРТОГОНАЛЬНЫМ НА РАВНОМЕРНОЙ СЕТКЕ}

\section{И.И. Шарапудинов}

Построено разложение дискретной функции в виде смешанного ряда по полиномам Чебышева. Получены оценки погрешности приближения функции и ее производных.

Библиография: 13 названий.

Эта работа посвящена теории смешанных рядов по полиномам Чебьшева $T_{n}^{\alpha, \beta}(x, N)$, $n=0,1, \ldots, N-1$, ортогональным на равномерной сетке $\Omega_{N}=\{0,1, \ldots, N-1\}$. Этиполиномы представляют собой дискретньй аналог полиномов Якоби $P_{n}^{\alpha, \beta}(x), n=0,1, \ldots$ Ранее в работах автора [1]-[9] были рассмотрены смешанные ряды по полиномам Лежандра, ультрасферическим полиномам и общим полиномам Якоби, а также полиномам Чебьшева $T_{n}^{\alpha, \beta}(x, N), n=0,1, \ldots, N-1$, при $\alpha=\beta=0$. Здесь рассматривается случай произвольных $\alpha, \beta>-1$. Основная идея введения смешанных рядов по ортогональным полиномам связана с задачей одновременного приближения функции и ее производных, которая, в свою очередь, возникает при численном решении дифференциальных уравнений самых различных типов. Дело в том, что, как отмечалось в цитированных работах, суммы Фурье по ортогональным полиномам для решения задачи одновременного приближения функции и ее нескольких производных не подходят. Это связано с тем, что вместе с ростом степени ортогонального полинома его производные (в дискретном случае - разностные производные) сильно растут вблизи концов интервала, на котором ортогональна рассматриваемая система полиномов. В частности, это касается и полиномов Чебышева $T_{n}^{\alpha, \beta}(x, N), n=0,1, \ldots, N-1$. Смешанные ряды по ортогональным полиномам возникли как альтернативный аппарат приближения функций, которьй позволяет эффективно решить задачу одновременного приближения функции и ее нескольких производных. Что касается рассматриваемых в настоящей работе смешанных рядов по полиномам Чебьшева $T_{n}^{\alpha, \beta}(x, N), n=0,1, \ldots, N-1$, то они естественньм образом возникают при решении задачи (которая чаше всего также связана с численным решением дифференциальных уравнений разностными методами) одновременного приближения дискретной функции, заданной на сетке $\Omega_{N}=\{0,1, \ldots, N-1\}$ и ее конечных разностей.

1. Некоторые свойства полиномов $T_{n}^{\alpha, \beta}(x, N)$. Для удобства ссылок мы здесь соберем из [10]-[12] некоторые формулы для полиномов Чебьшева $T_{n}^{\alpha, \beta}(x, N)$ и установим ряд вспомогательных утверждений. Пусть $N$ - натуральное число, $\alpha, \beta$ - произ- 
вольные комплексные числа. Положим

$$
\begin{gathered}
\rho(x)=\rho(x ; \alpha, \beta, N)=\frac{\Gamma(x+\beta+1) \Gamma(N-x+\alpha)}{\Gamma(x+1) \Gamma(N-x)}, \\
T_{n}^{\alpha, \beta}(x, N)=\frac{(-1)^{n}}{n !(N-1)^{[n]} \rho(x)} \Delta^{n}\left\{\rho(x)(x-N-\alpha)^{[n]} x^{[n]}\right\}
\end{gathered}
$$

где $\Delta^{n} f(x)$ - конечная разность $n$-го порядка функции $f(x)$ в точке $x$, т.е. $\Delta^{0} f(x)=f(x)$, $\Delta^{1} f(x)=\Delta f(x)=f(x+1)-f(x), \Delta^{n} f(x)=\Delta \Delta^{n-1} f(x), n \geqslant 1, a^{[0]}=1, a^{[k]}=a \times$ $(a-1) \cdots(a-k+1)$ при $k \geqslant 1$. Для каждого $0 \leqslant n \leqslant N-1$ равенство (1.2) определяет алгебраический полином степени $n$, для которого

$$
T_{n}^{\alpha, \beta}(N-1, N)=\left(\begin{array}{c}
n+\alpha \\
n
\end{array}\right), \quad T_{n}^{\alpha, \beta}(0, N)=(-1)^{n}\left(\begin{array}{c}
n+\beta \\
n
\end{array}\right) .
$$

Полиномы допускают следующее явное представление:

$$
T_{n}^{\alpha, \beta}(x, N)=(-1)^{n} \frac{\Gamma(n+\beta+1)}{n !} \sum_{k=0}^{n}(-1)^{k} \frac{n^{[k]}(n+\alpha+\beta+1)_{k} x^{[k]}}{\Gamma(k+\beta+1) k !(N-1)^{[k]}}
$$

Если $\alpha, \beta>-1$, то полиномы $T_{n}^{\alpha, \beta}(x, N), 0 \leqslant n \leqslant N-1$, образуют ортогональную с весом (1.1) систему на множестве $\Omega_{N}=\{0,1, \ldots, N-1\}$, точнее,

$$
\sum_{x \in \Omega_{N}} \mu(x) T_{n}^{\alpha, \beta}(x, N) T_{m}^{\alpha, \beta}(x, N)=h_{n, N}^{\alpha, \beta} \delta_{n m},
$$

где $\delta_{n m}-$ символ Кронекера,

$$
\begin{aligned}
\mu(x) & =\mu(x ; \alpha, \beta, N)=\frac{\Gamma(N) 2^{\alpha+\beta+1}}{\Gamma(N+\alpha+\beta+1)} \rho(x) \\
& =\frac{\Gamma(N) 2^{\alpha+\beta+1}}{\Gamma(N+\alpha+\beta+1)} \frac{\Gamma(x+\beta+1) \Gamma(N-x+\alpha)}{\Gamma(x+1) \Gamma(N-x)}, \\
h_{n, N}^{\alpha, \beta} & =\frac{(N+n+\alpha+\beta)^{[n]}}{(N-1)^{[n]}} \frac{\Gamma(n+\alpha+1) \Gamma(n+\beta+1) 2^{\alpha+\beta+1}}{n ! \Gamma(n+\alpha+\beta+1)(2 n+\alpha+\beta+1)} .
\end{aligned}
$$

При $n=0$ произведение $(\alpha+\beta+1) \Gamma(\alpha+\beta+1)$ следует заменить на $\Gamma(\alpha+\beta+2)$. Для $0 \leqslant n \leqslant N-1$ положим

$$
\tau_{n}^{\alpha, \beta}(x)=\tau_{n}^{\alpha, \beta}(x, N)=\left\{h_{n, N}^{\alpha, \beta}\right\}^{-1 / 2} T_{n}^{\alpha, \beta}(x, N) .
$$

Очевидно, если $0 \leqslant n, m \leqslant N-1$, то

$$
\sum_{x=0}^{N-1} \mu(x) \tau_{n}^{\alpha, \beta}(x, N) \tau_{m}^{\alpha, \beta}(x, N)=\delta_{n m}
$$

Другими словами, многочлены $\tau_{n}^{\alpha, \beta}(x, N), 0 \leqslant n \leqslant N-1$, образуют ортонормированную с весом $\mu(x)$ систему на $\{0,1, \ldots, N-1\}$. 
Формула Кристоффеля-Дарбу для многочленов Чебьшева $T_{n}^{\alpha, \beta}(x)=T_{n}^{\alpha, \beta}(x, N)$ имеет следуюший вид:

$$
\begin{aligned}
D_{n, N}^{\alpha, \beta}(x, y)= & \sum_{k=0}^{n} \frac{T_{k}^{\alpha, \beta}(x) T_{k}^{\alpha, \beta}(y)}{h_{k, N}^{\alpha, \beta}} \\
= & \frac{(N-1)^{[n+1]}}{(N+n+\alpha+\beta)^{[n]}} \frac{2^{-\alpha-\beta-1}}{2 n+\alpha+\beta+2} \frac{\Gamma(n+2) \Gamma(n+\alpha+\beta+2)}{\Gamma(n+\alpha+1) \Gamma(n+\beta+1)} \\
& \times \frac{T_{n+1}^{\alpha, \beta}(x) T_{n}^{\alpha, \beta}(y)-T_{n}^{\alpha, \beta}(x) T_{n+1}^{\alpha, \beta}(y)}{x-y} .
\end{aligned}
$$

Поскольку $\Delta a^{[k]}=k a^{[k-1]}$, из $(1.4)$ находим

$$
(n+1) T_{n+1}^{\alpha, \beta}(x, N)+(n+\beta+1) T_{n}^{\alpha, \beta}(x, N)=\frac{2 n+\alpha+\beta+2}{N-1} x T_{n}^{\alpha, \beta+1}(x-1, N-1) .
$$

Из равенства $\mu(N-1-x ; \beta, \alpha, N)=\mu(x ; \alpha, \beta, N)$, непосредственно вытекающего из (1.6), и соотношения ортогональности (1.5) следует, что при $\alpha, \beta>-1$

$$
T_{n}^{\alpha, \beta}(x, N)=(-1)^{n} T_{n}^{\beta, \alpha}(N-1-x, N) .
$$

Поскольку обе части этого равенства аналитичны относительно $\alpha$ и $\beta$, то оно справедливо для произвольных $\alpha$ и $\beta$. Из (1.11) и (1.12) имеем также следующее равенство:

$$
(n+\alpha+1) T_{n}^{\alpha, \beta}(x, N)-(n+1) T_{n+1}^{\alpha, \beta}(x, N)=\frac{2 n+\alpha+\beta+2}{N-1}(N-1-x) T_{n-1}^{\alpha+1, \beta}(x, N-1) .
$$

Полагая в (1.10) $y=N-1$, имеем

$$
\begin{aligned}
D_{n, N}^{\alpha, \beta}(x, N-1)= & \frac{T_{n}^{\alpha, \beta}(x)-\frac{n+1}{n+\alpha+1} T_{n+1}^{\alpha, \beta}(x)}{N-1-x} \\
& \times \frac{(N-1)^{[n+1]}}{(N+n+\alpha+\beta)^{[n]}} \frac{2^{-\alpha-\beta-1}}{2 n+\alpha+\beta+2} \frac{(n+\alpha+1) \Gamma(n+\alpha+\beta+2)}{\Gamma(\alpha+1) \Gamma(n+\beta+1)} \\
= & \frac{(N-2)^{[n]} \Gamma(n+\alpha+\beta+2) 2^{-\alpha-\beta-1}}{(N+n+\alpha+\beta)^{[n]} \Gamma(\alpha+1) \Gamma(n+\beta+1)} T_{n}^{\alpha+1, \beta}(x, N-1) .
\end{aligned}
$$

Последнее выражение является следствием равенства (1.13). Аналогично, полагая в (1.10) $y=0$ и учитывая (1.11), получаем

$$
D_{n, N}^{\alpha, \beta}(x, 0)=\frac{(N-2)^{[n]} \Gamma(n+\alpha+\beta+2) 2^{-\alpha-\beta-1}}{(N+n+\alpha+\beta)^{[n]} \Gamma(\beta+1) \Gamma(n+\alpha+1)} T_{n}^{\alpha, \beta+1}(x, N-1) .
$$

Непосредственно из явной формулы (1.4) мы можем вывести следующее полезное равенство:

$$
\Delta^{m} T_{n}^{\alpha, \beta}(x, N)=\frac{(n+\alpha+\beta+1)_{m}}{(N-1)^{[m]}} T_{n-m}^{\alpha+m, \beta+m}(x, N-m),
$$


где $(a)_{0}=1,(a)_{k}=a(a+1) \cdots(a+k-1)$ при $k \geqslant 1$. Если $\beta$ такое целое число, что $-n \leqslant \beta \leqslant-1$, то из (1.4) выводим также

$$
T_{n}^{\alpha, \beta}(x, N)=\frac{(n+\beta) !}{n !} \frac{(n+\alpha)^{[-\beta]} x^{[-\beta]}}{(N-1)^{[-\beta]}} T_{n+\beta}^{\alpha,-\beta}(x+\beta, N+\beta),
$$

а если $\alpha$ и $\beta$ целые, $-n \leqslant \beta \leqslant-1,-(n+\beta) \leqslant \alpha \leqslant-1, N \geqslant 2$, то

$$
T_{n}^{\alpha, \beta}(x, N)=\frac{(-1)^{\alpha} x^{[-\beta]}(N-x-1)^{[-\alpha]}}{(N-1)^{[-\beta]}(N-1+\beta)^{[-\alpha]}} T_{n+\alpha+\beta}^{-\alpha,-\beta}(x+\beta, N+\alpha+\beta) .
$$

Разностная формула Родрига допускает следующее обобщение:

$$
\begin{aligned}
\rho(x & +m ; \alpha, \beta, N+m) T_{n}^{\alpha, \beta}(x+m, N+m) \\
& =\frac{(-1)^{m}}{n^{[m]}(N)_{m}} \Delta^{m}\left\{\rho(x ; \alpha+m, \beta+m, N) T_{n-m}^{\alpha+m, \beta+m}(x, N)\right\},
\end{aligned}
$$

которое, впрочем, непосредственно вытекает из (1.2). Если в равенстве (1.16) мы заменим $\alpha, \beta$ и $n$ соответственно на $\alpha-m, \beta-m$ и $k+m$, то придем к формуле

$$
\Delta^{m} T_{k+m}^{\alpha-m, \beta-m}(x, N)=\frac{(k+\alpha+\beta)^{[m]}}{(N-1)^{[m]}} T_{k}^{\alpha, \beta}(x, N-m) .
$$

Пусть $a, \alpha>-1,0 \leqslant n \leqslant N-1$. Тогда [12]

$$
\begin{aligned}
\frac{T_{n}^{a, a}(x, N)}{T_{n}^{a, a}(N-1, N)}= & \sum_{j=0}^{[n / 2]} \frac{n !(\alpha+1)_{n-2 j}(n+2 a+1)_{n-2 j}(1 / 2)_{j}(a-\alpha)_{j}}{(n-2 j) !(2 j) !(a+1)_{n-2 j}(n-2 j+2 \alpha+1)_{n-2 j}} \\
& \times \frac{1}{(n-2 j+a+1)_{j}(n-2 j+\alpha+3 / 2)_{j}} \frac{T_{n-2 j}^{\alpha, \alpha}(x, N)}{T_{n-2 j}^{\alpha, \alpha}(N-1, N)}
\end{aligned}
$$

где $[n / 2]$ - целая часть числа $n / 2$.

Лемма 1.1. Пусть $\alpha>-1, k, r$ иелье, $r \geqslant 1, k \geqslant r+1$. Тогда

$$
T_{k+r}^{\alpha-r, \alpha-r}(x, N)=\sum_{j=0}^{r} \lambda_{j}^{\alpha} T_{k+r-2 j}^{\alpha, \alpha}(x, N)
$$

$2 \partial e$

$$
\lambda_{j}^{\alpha}=\lambda_{j}^{\alpha}(r, k)=\frac{(-1)^{j}(k-r+2 \alpha+1)_{k+r-2 j}(1 / 2)_{j} r^{[j]}(\alpha+k)^{[j]}}{(k+r-2 j+2 \alpha+1)_{k+r-2 j}(k+r-2 j+\alpha+3 / 2)_{j}(2 j) !} .
$$


ДокАЗАТЕЛЬСТво. Предположим сначала, что $\alpha-r>-1$; тогда, полагая $a=\alpha-r$, можем воспользоваться равенством (1.20). Это дает

$$
T_{k+r}^{\alpha-r, \alpha-r}(x, N)=\sum_{j=0}^{r} \lambda_{j}^{\alpha} T_{k+r-2 j}^{\alpha, \alpha}(x, N)
$$

где

$$
\begin{aligned}
\lambda_{j}^{\alpha}= & \frac{T_{k+r}^{\alpha-r, \alpha-r}(N-1, N)}{T_{k+r-2 j}^{\alpha, \alpha}(N-1, N)} \frac{(k+r) !(\alpha+1)_{k+r-2 j}(k-r+2 \alpha+1)_{k+r-2 j}}{(k+r-2 j) !(2 j) !(\alpha-r+1)_{k+r-2 j}} \\
& \times \frac{(1 / 2)_{j}(-r)_{j}}{(k+r-2 j+2 \alpha+1)_{k+r-2 j}(k-2 j+\alpha+1)_{j}(k+r-2 j+\alpha+3 / 2)_{j}} \\
= & \frac{(k-r+2 \alpha+1)_{k+r-2 j}(1 / 2)_{j}(-r)_{j}}{(k+r-2 j+2 \alpha+1)_{k+r-2 j}(k+r-2 j+\alpha+3 / 2)_{j}(2 j) !} \\
& \times \frac{(\alpha-r+1)_{k+r}}{(\alpha-r+1)_{k+r-2 j}(k-2 j+\alpha+1)_{j}} \\
= & \frac{(-1)^{j}(k-r+2 \alpha+1)_{k+r-2 j}(1 / 2)_{j} r^{[j]}(\alpha+k)^{[j]}}{(k+r-2 j+2 \alpha+1)_{k+r-2 j}(k+r-2 j+\alpha+3 / 2)_{j}(2 j) !} .
\end{aligned}
$$

Отсюда следует справедливость утверждения леммы 1.1 в случае $\alpha>r-1$. Нопоскольку $T_{k+r}^{\alpha-r, \alpha-r}(x, N), \lambda_{j}^{\alpha}$ и $T_{k+r-2 j}^{\alpha, \alpha}(x, N)$ представляют собой аналитические функции относительно $\alpha$, утверждение леммы 1.1 вытекает из уже доказанного случая.

ЛЕмма 1.2. Пусть $T_{n}(x, N)=T_{n}^{0,0}(x, N)$. Тогда для $0 \leqslant m \leqslant k, x \in \Omega_{N}$ имеет место равенство

$$
\frac{(x+m)^{[m]}}{(N+m)^{[m]}} \frac{(N-x)_{m}}{(N+m+1)_{m}} T_{k-m}^{m, m}(x, N)=\sum_{i=0}^{m}(-1)^{i} \sigma_{i}^{k} T_{k-m+2 i}(x, N),
$$

в котором при $k-m+2 i \leqslant N-1$

$$
\begin{aligned}
\sigma_{i}^{k}=\sigma_{i}^{k}(m, N)= & \frac{(N+m+k)^{[k-m]}(N+m-k-1)^{[2 i]}}{(N+k-m+2 i)^{[k-m+2 i]}} \\
& \times \frac{\left(k^{[m]}\right)^{2} m^{[i]}(1 / 2)_{i}(2 k+1-2 m+4 i)}{(2 i) !(2 k)^{[2 m-2 i]}(k-m+1)_{i}(k+3 / 2)_{i}(2 k+1)},
\end{aligned}
$$

а если $k-m+2 i>N-1, \operatorname{mo} \sigma_{i}^{k}(m, N)=0$.

ДокАЗАтЕльство. Выражение $(x+m)^{[m]}(N-x)_{m} T_{k-m}^{m, m}(x, N)$ представляет собой многочлен степени $k+m$; следовательно, мы можем записать $\left(x \in \Omega_{N}\right)$

$$
\frac{(x+m)^{[m]}}{(N+m)^{[m]}} \frac{(N-x)_{m}}{(N+m+1)_{m}} T_{k-m}^{m, m}(x, N)=\sum_{j=0}^{k+m} \gamma_{j} T_{j}(x, N),
$$

где

$$
\gamma_{j}=\frac{1}{h_{j, N}^{0,0}} \frac{2}{N} \sum_{t=0}^{N-1} \frac{(t+m)^{[m]}}{(N+m)^{[m]}} \frac{(N-t)_{m}}{(N+m+1)_{m}} T_{k-m}^{m, m}(t, N) T_{j}(t, N),
$$


если $0 \leqslant j \leqslant N-1$ и $\gamma_{j}=0$ при $j \geqslant N$. Поскольку многочлен $T_{k-m}^{m, m}(t, N)$ при $j<k-m$ ортогонален с весом $(x+m)^{[m]}(N-x)_{m} \mathrm{~K} T_{j}(x, N)$, то соответствуюшие $\gamma_{j}=0$. Кроме того, сумма (1.24) обращается в нуль, если $k-m$ и $j$ не являются одновременно четными или нечетньми. Следовательно, (1.23) можно переписать так:

$$
\frac{(x+m)^{[m]}}{(N+m)^{[m]}} \frac{(N-x)_{m}}{(N+m+1)_{m}} T_{k-m}^{m, m}(x, N)=\sum_{i=0}^{m} \gamma_{k-m+2 i} T_{k-m+2 i}(x, N),
$$

где

$$
\gamma_{k-m+2 i}=\frac{1}{h_{k-m+2 i, N}^{0,0}} \frac{2}{N} \sum_{t=0}^{N-1} \frac{(t+m)^{[m]}}{(N+m)^{[m]}} \frac{(N-t)_{m}}{(N+m+1)_{m}} T_{k-m}^{m, m}(t, N) T_{k-m+2 i}(t, N) ;
$$

в частности, если $k-m+2 i>N-1$, то $\gamma_{k-m+2 i}=0$. Для вычисления суммы (1.26) при $k-m+2 i \leqslant N-1$ воспользуемся леммой 1.1. Полагая $a=0, \alpha=m, n=k-m+2 i$ и учитьвая равенство

$$
T_{n}^{\alpha, \beta}(N-1, N)=\left(\begin{array}{c}
n+\alpha \\
n
\end{array}\right)
$$

из леммы 1.1 находим

$$
\begin{aligned}
T_{k-m+2 i}(x, N)= & \sum_{j=0}^{q} \frac{(2 k-2 m+4 i-2 j) !(k+m+2 i-2 j) !}{(k-m+2 i-2 j) !(2 j) !(2 k+4 i-4 j) !} \\
& \times \frac{(1 / 2)_{j} m^{[j]}(-1)^{j}}{(k-m+2 i-2 j+1)_{j}(k+2 i-2 j+3 / 2)_{j}} T_{k-m+2 i-2 j}^{m, m}(x, N),
\end{aligned}
$$

где $q=\min \{m,[(k-m+2 i) / 2]\}$. Умножим обе части этого равенства на $\mu(x, m, m, N) \times$ $T_{k-m}^{m, m}(x, N)$ и просуммируем от 0 до $N-1$; тогда

$$
\begin{gathered}
\frac{2^{2 m+1}}{N} \sum_{t=0}^{N-1} \frac{(t+m)^{[m]}}{(N+m)^{[m]}} \frac{(N-t)_{m}}{(N+m+1)_{m}} T_{k-m}^{m, m}(t, N) T_{k-m+2 i}(t, N) \\
=\frac{(2 k-2 m+2 i) !(k+m) !(1 / 2)_{i} m^{[i]}(-1)^{i}}{(k-m) !(2 i) !(2 k) !(k-m+1)_{i}(k+3 / 2)_{i}} h_{k-m, N}^{m, m} .
\end{gathered}
$$

Из (1.26) и (1.27) имеем

$$
\gamma_{k-m+2 i}=\frac{h_{k-m, N}^{m, m}}{2^{2 m} h_{k-m+2 i, N}^{0,0}} \frac{(2 k-2 m+2 i) !(k+m) !(1 / 2)_{i} m^{[i]}(-1)^{i}}{(k-m) !(2 i) !(2 k) !(k-m+1)_{i}(k+3 / 2)_{i}} .
$$

Если мы воспользуемся равенством (1.7), то с учетом (1.22) мы можем переписать (1.28) так:

$$
\begin{aligned}
\gamma_{k-m+2 i}= & \frac{(-1)^{i}(N+m+k)^{[k-m]}(N-1)^{[k-m+2 i]}}{(N-1)^{[k-m]}(N+k-m+2 i)^{[k-m+2 i]}} \\
& \times \frac{k !^{2}(2 k-2 m+4 i+1)}{(2 k+1)(k-m) !(k+m) !} \frac{(2 k-2 m+2 i) !(k+m) ! m^{[i]}(1 / 2)_{i}}{(2 k) !(k-m) !(2 i) !(k-m+1)_{i}(k+3 / 2)_{i}} \\
= & (-1)^{i} \frac{(N+m+k)^{[k-m]}(N+m-k-1)^{[2 i]}}{(N+k-2 m+2 i)^{[k-m+2 i]}} \\
& \times \frac{\left(k^{[m]}\right)^{2} m^{[i]}(1 / 2)_{i}(2 k+1-2 m+4 i)}{(2 i) !(2 k)^{[2 m-2 i]}(k-m+1)_{i}(k+3 / 2)_{i}(2 k+1)} \\
= & (-1)^{i} \sigma_{i}^{k}(m, N) .
\end{aligned}
$$


Подставляя это значение в (1.25), приходим к утверждению леммы 1.2.

При исследовании вопросов сходимости дискретных рядов Фурье-Чебышева важную роль играют асимптотические свойства полиномов $T_{n}^{\alpha, \beta}(x)$ при $n, N \rightarrow \infty$. Пусть $a>0,0 \leqslant n \leqslant a N^{1 / 2}, 0<\varepsilon<1, \alpha, \beta$-произвольные вешественные числа. Тогда имеет место [10] следующая асимптотическая формула:

$$
T_{n}^{\alpha, \beta}\left[\frac{N-1}{2}(1+t), N\right]=P_{n}^{\alpha, \beta}(t)+V_{n, N}^{\alpha, \beta}(t),
$$

для остаточного члена $V_{n, N}^{\alpha, \beta}(t)$ которой справедлива оценка

$$
\left|V_{n, N}^{\alpha, \beta}(t)\right| \leqslant c(\alpha, \beta, a, \varepsilon) \frac{n^{3 / 2}}{N}, \quad-1+\varepsilon \leqslant t \leqslant 1-\varepsilon .
$$

Вблизи точек -1 и 1 для остаточного члена $V_{n, N}^{\alpha, \beta}(t)$ справедливы оценки

$$
\begin{array}{ll}
\left|V_{n, N}^{\alpha, \beta}(t)\right| \leqslant c(\alpha, \beta, a, q) \frac{n^{2+\beta}}{N}, & |1+t| \leqslant q n^{-2} \\
\left|V_{n, N}^{\alpha, \beta}(t)\right| \leqslant c(\alpha, \beta, a, q) \frac{n^{2+\alpha}}{N}, & |1-t| \leqslant q n^{-2} .
\end{array}
$$

2. Дискретное преобразование Фурье-Чебышева. Пусть $\alpha, \beta>-1, \mu(x)=$ $\mu(x ; \alpha, \beta, N)$ - функция (1.6). Для двух произвольньх дискретных функций $d$ и $q$, заданных на множестве $\Omega_{N}=\{0,1, \ldots, N-1\}$ определим скалярное произведение

$$
(f, g)_{\mu}=\sum_{x \in \Omega_{N}} \mu(x) d(x) q(x) .
$$

Евклидово пространство всех дискретных функций вида $d: \Omega_{N} \rightarrow \mathbb{R}$, в котором скалярное произведение определено с помошью равенства $(2.1)$ обозначим через $\ell_{2, \mu}^{N}$. Если $d \in \ell_{2, \mu}^{N}$, то через $\|d\|_{\mu, N}$ обозначим норму функции $d$, т.е.

$$
\|d\|_{\mu, N}=\left(\sum_{x \in \Omega_{N}} \mu(x) d^{2}(x)\right)^{1 / 2}
$$

Для $d \in \ell_{2, \mu}^{N}$ мы можем определить коэффициенты Фурье-Чебышева

$$
d_{k}^{\alpha, \beta}=d_{k}^{\alpha, \beta}(N)=\sum_{t \in \Omega_{N}} \mu(t) \tau_{k}^{\alpha, \beta}(t, N) d(t), \quad k \in \Omega_{N}
$$

Поскольку система $\left\{\tau_{k}^{\alpha, \beta}(t, N)\right\}_{0}^{N-1}$ ортонормирована на $\Omega_{N}$ с весом $\mu(x)(\alpha, \beta>-1)$, то

$$
d(x)=\sum_{k=0}^{N-1} d_{k}^{\alpha, \beta} \tau_{k}^{\alpha, \beta}(x, N), \quad x \in \Omega_{N} .
$$


Равенства (2.3) определяют прямое дискретноепреобразование Фурье-Чебышева, а равенства (2.4) определяют обратное преобразование Фурье-Чебьшева. Рассмотрим частичную сумму ряда $(2.4)$ порядка $n \leqslant N-1$

$$
S_{n, N}^{\alpha, \beta}(d)=S_{n, N}^{\alpha, \beta}(d, x)=\sum_{k=0}^{n} d_{k}^{\alpha, \beta} \tau_{k}^{\alpha, \beta}(x, N) .
$$

Она доставляет функции $d=d(x)$ наилучшее приближение в пространстве $\ell_{2, \mu}^{N}$ среди всех алгебраических полиномов степени $n$, т.е.

$$
\left\|d-S_{n, N}^{\alpha, \beta}(d)\right\|_{\ell_{2, \mu}^{N}}=\left(\sum_{t \in \Omega_{N}}\left(d(x)-S_{n, N}^{\alpha, \beta}(d, x)\right)^{2} \mu(x)\right)^{1 / 2}=E_{n}(d)_{\ell_{2, \mu}^{N}}=\inf _{P_{n}}\left\|d-P_{n}\right\|_{\ell_{2, \mu}^{N}},
$$

где нижняя грань берется по всем алгебраическим полиномам $P_{n}(x)$ степени $n$. Из $(2.4)$ следует, что если $n \geqslant N-1$, то $E_{n}(d)_{\ell_{2, \mu}^{N}}=0$. Если $p_{n}=p_{n}(x)-$ произвольньй алгебраический полином степени $n \leqslant N-1$, то из (2.6) следует, что

$$
S_{n, N}^{\alpha, \beta}\left(p_{n}, x\right)=p_{n}(x), \quad x \in \mathbb{R} .
$$

Далее отметим, что равенство Парсеваля для системы полиномов Чебьшева $\left\{\tau_{k}^{\alpha, \beta}(x\right.$, $N)\}_{k=0}^{N-1}$ приобретает следуюший вид:

$$
\sum_{t \in \Omega_{N}}\left(d(x)-S_{n, N}^{\alpha, \beta}(d, x)\right)^{2} \mu(x)=\sum_{k=n+1}^{N-1}\left(d_{k}^{\alpha, \beta}\right)^{2}
$$

Сопоставляя (2.6) и (2.8), имеем также

$$
E_{n}(d)_{\ell_{2, \mu}^{N}}=\left(\sum_{k=n+1}^{N-1}\left(d_{k}^{\alpha, \beta}\right)^{2}\right)^{1 / 2}
$$

Если мы воспользуемся ядром $D_{n, N}^{\alpha, \alpha}(x, t)$, то равенство $(2.5)$ можно переписать следуюшим образом:

$$
S_{n, N}^{\alpha, \beta}(d, x)=\sum_{t=0}^{N-1} D_{n, N}^{\alpha, \alpha}(x, t) d(t) \mu(t)
$$

В частности, если $x=0$, то из (1.15) и (2.10) получим

$$
S_{n, N}^{\alpha, \beta}(d, 0)=\frac{(N-2)^{[n]} \Gamma(n+\alpha+\beta+2) 2^{-\alpha-\beta-1}}{(N+n+\alpha+\beta)^{[n]} \Gamma(\beta+1) \Gamma(n+\alpha+1)} \sum_{t=0}^{N-1} \mu(t) T_{n}^{\alpha, \beta+1}(t, N-1) d(t) .
$$

Рассмотрим $S_{n, N}^{\alpha, \beta}(d, 0)$ как линейньй функционал, определенный в пространстве $C\left(\Omega_{N}\right)$, состоящем из дискретных функций вида $d: \Omega_{N} \rightarrow \mathbb{R}$, для которых норма определяется следуюшим образом:

$$
\|d\|_{C\left(\Omega_{N}\right)}=\max _{x \in \Omega_{N}}|d(x)|
$$


Пусть

$$
\left\|S_{n, N}^{\alpha, \beta}(0)\right\|_{C\left(\Omega_{N}\right)}=\sup _{\|d\|_{C(\Omega)_{N}} \leqslant 1}\left|S_{n, N}^{\alpha, \beta}(d, 0)\right|
$$

- норма функционала $S_{n, N}^{\alpha, \beta}(0): C\left(\Omega_{N}\right) \rightarrow \mathbb{R}$. Из (2.11)-(2.13) нетрудно увидеть, что $\left\|S_{n, N}^{\alpha, \beta}(0)\right\|_{C\left(\Omega_{N}\right)}=\frac{(N-2)^{[n]} \Gamma(n+\alpha+\beta+2) 2^{-\alpha-\beta-1}}{(N+n+\alpha+\beta)^{[n]} \Gamma(\beta+1) \Gamma(n+\alpha+1)} \sum_{t=0}^{N-1} \mu(t)\left|T_{n}^{\alpha, \beta+1}(t, N-1)\right|$.

Лемма 2.1. Пусть $\alpha>-1, \beta>-1 / 2, a>0,0 \leqslant n \leqslant a N^{1 / 2}$. Тогда

$$
\left\|S_{n, N}^{\alpha, \beta}(0)\right\|_{C\left(\Omega_{N}\right)} \geqslant c(\alpha, \beta, a) n^{\beta+1 / 2}\left(1-c_{1}(\alpha, \beta, a) \frac{n^{2}}{N}\right) .
$$

ДоКАЗАТЕЛЬСТВО. Воспользуемся асимптотической формулой (1.29); тогда

$$
T_{n}^{\alpha, \beta}\left[\frac{N-2}{2}(1+x), N-1\right]=P_{n}^{\alpha, \beta+1}(x)+V_{n, N-1}^{\alpha, \beta+1}(x),
$$

где для остаточного члена $V_{n, N-1}^{\alpha, \beta+1}(x)$ справедлива оценка

$$
\left|V_{n, N-1}^{\alpha, \beta+1}(t)\right| \leqslant c(\alpha, \beta, a, \varepsilon) \frac{n^{3 / 2}}{N}, \quad-1+\varepsilon \leqslant t \leqslant 1-\varepsilon .
$$

Пусть

$$
k(\theta)=\pi^{-1 / 2}\left(\sin \frac{\theta}{2}\right)^{-\alpha-1 / 2}\left(\cos \frac{\theta}{2}\right)^{-\beta-1 / 2}, \lambda=n+\frac{\alpha+\beta+1}{2}, \gamma=-\left(\frac{\alpha+1}{2}\right) \frac{\pi}{2} .
$$

Тогда при $0<\theta<\pi$ имеет место [13] следующая асимптотическая формула:

$$
P_{n}^{\alpha, \beta}(\cos \theta)=n^{-1 / 2} k(\theta)\left\{\cos (\lambda \theta+\gamma)+\frac{r_{n}(\theta)}{n \sin \theta}\right\}
$$

для остаточного члена $r_{n}(\theta)=r_{n}(\theta ; \alpha, \beta)$ которой имеет место оценка

$$
\left|r_{n}(\theta)\right| \leqslant c(\alpha, \beta, \delta), \quad 0<\frac{\delta}{n} \leqslant \theta \leqslant \pi-\frac{\delta}{n} .
$$

Сопоставляя (2.15)-(2.18), замечаем, что

$$
T_{n}^{\alpha, \beta}\left[\frac{N-2}{2}(1+x), N-1\right]=n^{-1 / 2} k(\theta) \cos (\lambda \theta+\gamma)+U_{n, N}^{\alpha, \beta}(\theta),
$$

где $x=\cos \theta$,

$$
\left|U_{n, N}^{\alpha, \beta}(\theta)\right| \leqslant c(\alpha, \beta, a, \varepsilon)\left(n^{-3 / 2}+\frac{n^{3 / 2}}{N}\right), \quad \arccos (\varepsilon) \leqslant \theta \leqslant \arccos (\pi-\varepsilon) .
$$


Далее заметим, что из (1.6) и формулы Стирлинга вытекает следующая оценка (здесь $\arccos (\varepsilon) \leqslant \theta \leqslant \arccos (\pi-\varepsilon))$ :

$$
\frac{c_{1}(\alpha, \beta, \varepsilon)}{N} \leqslant \mu\left[\frac{N-2}{2}(1+\cos \theta), \alpha, \beta, N\right] \leqslant \frac{c_{2}(\alpha, \beta, \varepsilon)}{N} .
$$

Пусть $x_{t}=-1+2 t /(N-2), \theta_{t}=\arccos x_{t}$. Тогда из $(2.19)-(2.21)$ вьводим

$$
\begin{aligned}
\sum_{t=0}^{N-1} & \mu(t)\left|T_{n}^{\alpha, \beta+1}(t, N-1)\right| \\
= & \sum_{t=0}^{N-1} \mu\left[\frac{N-2}{2}\left(1+x_{t}\right), \alpha, \beta, N\right]\left|T_{n}^{\alpha, \beta}\left[\frac{N-2}{2}\left(1+x_{t}\right), N-1\right]\right| \\
> & \sum_{-1+\varepsilon \leqslant x_{t} \leqslant 1-\varepsilon} n^{-1 / 2} k(\theta)|\cos (\lambda \theta+\gamma)| \mu\left[\frac{N-2}{2}\left(1+\cos \theta_{t}\right), \alpha, \beta, N\right] \\
& -\sum_{-1+\varepsilon \leqslant x_{t} \leqslant 1-\varepsilon}\left|U_{n, N}^{\alpha, \beta}\left(\theta_{t}\right)\right| \mu\left[\frac{N-2}{2}\left(1+\cos \theta_{t}\right), \alpha, \beta, N\right] \\
\geqslant & \frac{c_{1}(\alpha, \beta, \varepsilon)}{N n^{1 / 2}}-\sum_{-1+\varepsilon \leqslant x_{t} \leqslant 1-\varepsilon} k\left(\theta_{t}\right)\left|\cos \left(\lambda \theta_{t}+\gamma\right)\right|-c(\alpha, \beta, a, \varepsilon)\left(n^{-3 / 2}+\frac{n^{3 / 2}}{N}\right) \\
\geqslant & c(\alpha, \beta, a, \varepsilon)\left(n^{-1 / 2}-\frac{n^{3 / 2}}{N}\right) .
\end{aligned}
$$

Осталось заметить, что если $n \leqslant a N^{1 / 2}$, то

$$
\begin{aligned}
\frac{(N-2)^{[n]}}{(N+n+\alpha+\beta)^{[n]}} & \geqslant \frac{(N-n)^{n}}{(N+n+\alpha+\beta)^{n}}=\left(1-\frac{2 n+\alpha+\beta}{N+n+\alpha+\beta}\right)^{n} \\
& =\left(1-\frac{2 n+\alpha+\beta}{N+n+\alpha+\beta}\right)^{\frac{N+n+\alpha+\beta}{2 n+\alpha+\beta} \frac{n(2 n+\alpha+\beta)}{N+n+\alpha+\beta}} \\
& >e^{-\frac{n(2 n+\alpha+\beta)}{N+n+\alpha+\beta}}>c(a, \alpha, \beta)>0 .
\end{aligned}
$$

Утверждение леммы 2.1 вытекает из (2.14), (2.22) и (2.23).

Лемма 2.1 показывает, что если $\beta>-1 / 2$, то норма функционала $S_{n, N}^{\alpha, \beta}=S_{n, N}^{\alpha, \beta}(d, 0)$ растет вместе с $n$ со скоростью $n^{\beta+1 / 2}$ и, как следствие, оператор частичных сумм $S_{n, N}^{\alpha, \beta}=S_{n, N}^{\alpha, \beta}(d)$, вообще говоря, не является хорошим средством приближения дискретных функций $d \in C\left(\Omega_{N}\right)$, а именно: вблизи точки $x=0$ приближение функции $d=d(x)$, доставляемое частичной суммой $S_{n, N}^{\alpha, \beta}(d, x)$, может оказаться в $n^{\beta+1 / 2}$ раз хуже по порядку, чем наилучшее приближение функции $d$ в метрике пространства $C\left(\Omega_{N}\right)$ алгебраическими полиномами степени $n$. Ситуация ухудшится еще сильнее, если мы предпримем попытку приблизить конечные разности $\Delta^{\nu} d(x)$ функции $d$ конечными разностями $\Delta^{\nu} S_{n, N}^{\alpha, \beta}(d, x)$. Это обстоятельство побудило ввести новые смешанные $р$ яды по полиномам Чебьшева $T_{n}^{\alpha, \beta}(x, M)$, конструкция которых столь же проста как и у рядов Фурье по этим полиномам, а их частичные суммы успешно могут быть использованы в задаче одновременного приближения дискретных функций и их конечных разностей. 
3. Смешанные ряды по полиномам $T_{n}^{\alpha, \beta}(x, M)$. Пусть $r$ и $N$-натуральные числа. Рассмотрим дискретную функцию $d(x)$, заданную на сетке $\bar{\Omega}_{N+2 r}=\{-r,-r+1, \ldots$, $-1,0,1, \ldots, N-1, N, \ldots, N-1+r\}$. Положим

$$
\begin{gathered}
F(x)=d(x-r), \quad x \in \Omega_{N+2 r} \\
b(x)=b(x ; r, N)=\Delta^{r} F(x) .
\end{gathered}
$$

Дискретная функция $b(x)$ определена на сетке $\Omega_{N+r}$ и, следовательно, ее можно разложить в конечньй ряд Фурье по ортонормированной системе полиномов Чебьшева $\left\{\tau_{k}^{\alpha, \beta}(x, N+r)\right\}_{k=0}^{N-1+r}$

$$
b(x)=\sum_{k=0}^{N-1+r} d_{r, k}^{\alpha, \beta} \tau_{k}^{\alpha, \beta}(x, N+r)
$$

где $\alpha, \beta>-1$,

$$
d_{r, k}^{\alpha, \beta}=d_{r, k}^{\alpha, \beta}(N+r)=\sum_{t \in \Omega_{N+r}} \mu(t) b(t) \tau_{k}^{\alpha, \beta}(t, N+r) .
$$

Далее, запишем дискретный аналог формулы Тейлора $\left(x \in \Omega_{N+r}\right)$

$$
\begin{aligned}
F(x+1)= & F(0)+\frac{\Delta F(0)}{1 !}(x+1)+\frac{\Delta^{2} F(0)}{2 !}(x+1)^{[2]}+\cdots \\
& +\frac{\Delta^{r-1} F(0)}{(r-1) !}(x+1)^{[r-1]}+\frac{1}{(r-1) !} \sum_{t=0}^{x}(x-t)^{[r-1]} \Delta^{r} F(t)
\end{aligned}
$$

и подставим здесь вместо выражения $\Delta^{r} F(t)$ его значение, найденное из (3.2) и (3.3); тогда

$$
\begin{aligned}
F(x+1)= & F(0)+\frac{\Delta F(0)}{1 !}(x+1)+\cdots+\frac{\Delta^{r-1} F(0)}{(r-1) !}(x+1)^{[r-1]} \\
& +\frac{1}{(r-1) !} \sum_{k=0}^{N-1+r} d_{r, k}^{\alpha, \beta} \sum_{t=0}^{x}(x-t)^{[r-1]} \tau_{k}^{\alpha, \beta}(t, N+r) .
\end{aligned}
$$

Учитьвая равенство (1.8), мы можем переписать (3.6) еше так:

$$
\begin{aligned}
F(x+1)= & F(0)+\frac{\Delta F(0)}{1 !}(x+1)+\cdots+\frac{\Delta^{r-1} F(0)}{(r-1) !}(x+1)^{[r-1]} \\
& +\sum_{k=0}^{N-1+r} \frac{d_{r, k}^{\alpha, \beta}}{\left\{h_{k, N+r}^{\alpha, \beta}\right\}^{1 / 2}} \frac{1}{(r-1) !} \sum_{t=0}^{x}(x-t)^{[r-1]} T_{k}^{\alpha, \beta}(t, N+r) .
\end{aligned}
$$

Пусть $\lambda=\alpha+\beta,(k+\lambda)^{[r]} \neq 0$. Тогда мы можем воспользоваться равенством (1.19). Это дает

$$
T_{k}^{\alpha, \beta}(t, N+r)=\frac{(N-1+2 r)^{[r]}}{(k+\lambda)^{[r]}} \Delta^{r} T_{k+r}^{\alpha-r, \beta-r}(t, N+2 r) .
$$


С другой стороны, из формулы (3.5), примененной к функции $T_{k+r}^{\alpha-r, \beta-r}(t, N+2 r)$ имеем

$$
\begin{aligned}
& \frac{1}{(r-1) !} \sum_{t=0}^{x}(x-t)^{[r-1]} \Delta^{r} T_{k+r}^{\alpha-r, \beta-r}(t, N+2 r) \\
& \quad=T_{k+r}^{\alpha-r, \beta-r}(x+1, N+2 r)-\sum_{\nu=0}^{r-1} \frac{\Delta^{\nu} T_{k+r}^{\alpha-r, \beta-r}(0, N+2 r)}{\nu !}(x+1)^{[\nu]} .
\end{aligned}
$$

Далее, в силу (1.16)

$$
\Delta^{\nu} T_{k+r}^{\alpha-r, \beta-r}(0, N+2 r)=\frac{(k+\lambda-r+1)_{\nu}}{(N-1+2 r)^{[\nu]}} T_{k+r-\nu}^{\alpha-r+\nu, \beta-r+\nu}(0, N+2 r-\nu),
$$

а из (1.3) имеем

$$
T_{k+r-\nu}^{\alpha-r+\nu, \beta-r+\nu}(0, N+2 r-\nu)=(-1)^{k+r-\nu}\left(\begin{array}{c}
k+\beta \\
k+r-\nu
\end{array}\right)=\frac{(-1)^{k+r-\nu} \Gamma(k+\beta+1)}{\Gamma(\nu-r+\beta+1)(k+r-\nu) !} .
$$

Сопоставляя (3.10) и (3.11), находим

$$
\Delta^{\nu} T_{k+r}^{\alpha-r, \beta-r}(0, N+2 r)=\frac{(-1)^{k+r-\nu} \Gamma(k+\beta+1)(k+\lambda-r+1)_{\nu}}{\Gamma(\nu-r+\beta+1)(k+r-\nu) !(N-1+2 r)^{[\nu]}}
$$

В дальнейшем мы отдельно рассмотрим два случая:

1) $-2<\lambda$ не целое;

2) $\lambda \in\{-1,0,1\}$.

Пусть $\lambda$ не целое; тогда каково бы ни было $k=0,1, \ldots$ выражение $(k+\lambda)^{[r]}$ не обращается в нуль и, следовательно, мы можем воспользоваться равенством (3.8). Это в сочетании с $(3.9)$ и $(3.12)$ дает

$$
\begin{aligned}
& \frac{1}{(r-1) !} \sum_{t=0}^{x}(x-t)^{[r-1]} T_{k}^{\alpha, \beta}(t, N+r) \\
& \quad=\frac{(N-1+2 r)^{[r]}}{(k+\lambda)^{[r]}} T_{k+r}^{\alpha-r, \beta-r}(x+1, N+2 r) \\
& \quad-\frac{(N-1+2 r)^{[r]}}{(k+\lambda)^{[r]}} \sum_{\nu=0}^{r-1} \frac{(-1)^{k+r-\nu} \Gamma(k+\beta+1)(k+\lambda-r+1)_{\nu}(x+1)^{[\nu]}}{\nu ! \Gamma(\nu-r+\beta+1)(k+r-\nu) !(N-1+2 r)^{[\nu]}} .
\end{aligned}
$$

Сопоставляя $(3.1),(3.7)$ и (3.13), получим

$$
d(x-r)=Q_{r-1, N}(d, x)+A_{r-1, N}^{\alpha, \beta}(d, x)+\mathscr{F}_{r, N}^{\alpha, \beta}(d, x),
$$


где $x \in \Omega_{N+r}$,

$$
\begin{aligned}
Q_{r-1, N}(d, x)= & d(-r)+\frac{\Delta d(-r)}{1 !} x+\cdots+\frac{\Delta^{r-1} d(-r)}{(r-1) !} x^{[r-1]} \\
A_{r-1, N}^{\alpha, \beta}(d, x)= & -\sum_{\nu=0}^{r-1}\left(\frac{(-1)^{r-\nu}(N+r)_{r-\nu}}{\nu ! \Gamma(\nu-r+\beta+1)}\right. \\
& \left.\times \sum_{k=0}^{N-1+r} \frac{(-1)^{k} d_{r, k}^{\alpha, \beta}}{\left\{h_{k, N+r}^{\alpha, \beta}\right\}^{1 / 2}} \frac{\Gamma(k+\beta+1)(k+\lambda-r+1)_{\nu}}{(k+r-\nu) !(k+\lambda)^{[r]}}\right) x^{[\nu]} \\
\mathscr{F}_{r, N}^{\alpha, \beta}(d, x)= & (N-1+2 r)^{[r]} \sum_{k=0}^{N-1+r} \frac{d_{r, k}^{\alpha, \beta}}{\left\{h_{k, N+r}^{\alpha, \beta}\right\}^{1 / 2}} \frac{T_{k+r}^{\alpha-r-\beta}(x, N+2 r)}{(k+\lambda)^{[r]}} .
\end{aligned}
$$

Правую часть равенства $(3.14)$, а также функцию $\mathscr{F}_{r, N}^{\alpha, \beta}(x)=\mathscr{F}_{r, N}^{\alpha, \beta}(d, x)$ будем назьвать смешанны.ми рядами по полиномам Чебьшева $T_{k}^{\alpha, \beta}(x, M)$. Покажем, что если $r \leqslant N$, то равенство (3.14) останется в силе для всех $x \in \Omega_{N+2 r}$, а не только для $x \in \Omega_{N+r}$. В самом деле, рассмотрим функцию $\bar{d}(x)=d(N-1-x), x \in \bar{\Omega}_{N+2 r}$. Тогда, полагая $\bar{F}(x)=\bar{d}(x-r)=d(N-1-x+r)=F(N-1+2 r-x)$, имеем

$$
\begin{aligned}
\Delta^{r} \bar{F}(t) & =\sum_{l=0}^{r}(-1)^{r+l}\left(\begin{array}{l}
r \\
l
\end{array}\right) \bar{F}(t+l)=\sum_{l=0}^{r}(-1)^{r+l}\left(\begin{array}{l}
r \\
l
\end{array}\right) F(N-1+2 r-l-t) \\
& =\sum_{\nu=0}^{r}(-1)^{2 r-\nu}\left(\begin{array}{c}
r \\
r-\nu
\end{array}\right) F(N-l+r+\nu-t) \\
& =(-1)^{r} \sum_{\nu=0}^{r}(-1)^{r+\nu}\left(\begin{array}{l}
r \\
\nu
\end{array}\right) F(N-1+r-t+\nu)=(-1)^{r} \Delta^{r} F(N-1+r-t)
\end{aligned}
$$

и, стало быть,

$$
\bar{b}(t)=\Delta^{r} \bar{F}(t)=(-1)^{r} \Delta^{r} F(N-1+r-t)=(-1)^{r} b(N-1+r-t) .
$$

Поэтому в силу симметрии (1.12) имеем

$$
\begin{aligned}
& \bar{b}_{k}^{\beta, \alpha}(N+R)= \sum_{t \in \Omega_{N+r}} \mu(t ; \beta, \alpha, N+r) \tau_{k}^{\beta, \alpha}(t, N+r) \bar{b}(t) \\
&=(-1)^{r} \sum_{t \in \Omega_{N+r}} \mu(t ; \beta, \alpha, N+r) \tau_{k}^{\beta, \alpha}(t, N+r) b(N-1+r-t) \\
&=(-1)^{r+k} \sum_{t \in \Omega_{N+r}} \mu(N-1+r-t ; \alpha, \beta, N+r) \\
& \times \tau_{k}^{\alpha, \beta}(N-1+r-t, N+r) b(N-1+r-t) \\
&=(-1)^{r+k} b_{k}^{\alpha, \beta}(N+r), \\
& \frac{(-1)^{r+k}}{\left\{h_{k, N}^{\beta, \alpha}\right\}^{1 / 2}} T_{k+r}^{\beta-r, \alpha-r}(x, N+2 r)=\frac{1}{\left\{h_{k, N}^{\alpha, \beta}\right\}^{1 / 2}} T_{k+r}^{\alpha-r, \beta-r}(N-1+2 r-x, N+2 r) .
\end{aligned}
$$


Отсюда и из (3.25) вьводим

$$
\mathscr{F}_{r, N}^{\alpha, \beta}(d, N-1+2 r-x)=\mathscr{F}_{r, N}^{\beta, \alpha}(\bar{d}, x)
$$

Докажем теперь, что если $r \leqslant N$, то равенство (3.14) справедливо при всех $x \in \Omega_{N+2 r}$. С этой целью положим

$$
p_{r-1, N}^{\alpha, \beta}(x)=Q_{r-1, N}(d, x)+A_{r-1, N}^{\alpha, \beta}(d, x)
$$

и перепишем равенство (3.14) так:

$$
d(x-r)=p_{r-1, N}^{\alpha, \beta}(d, x)+\mathscr{F}_{r, N}^{\alpha, \beta}(d, x), \quad x \in \Omega_{N+r},
$$

и, аналогично,

$$
\bar{d}(x-r)=p_{r-1, N}^{\beta, \alpha}(\bar{d}, x)+\mathscr{F}_{r, N}^{\beta, \alpha}(\bar{d}, x), \quad x \in \Omega_{N+r} .
$$

Равенство (3.22) с учетом (3.19) принимает следующий вид:

$$
\begin{aligned}
d(N-1+r-x) & =p_{r-1, N}^{\beta, \alpha}(\bar{d}, x)+\mathscr{F}_{r, N}^{\beta, \alpha}(\bar{d}, x) \\
& =p_{r-1, N}^{\beta, \alpha}(\bar{d}, x)+\mathscr{F}_{r, N}^{\alpha, \beta}(d, N-1+2 r-x), \quad x \in \Omega_{N+r}
\end{aligned}
$$

или, что то же самое,

$$
d(x-r)=p_{r-1, N}^{\beta, \alpha}(\bar{d}, N-1+2 r-x)+\mathscr{F}_{r, N}^{\alpha, \beta}(d, x)
$$

где $N-1+2 r-x \in \Omega_{N+r}$, т.е. $x \in\{r, r+1, \ldots, N-1+2 r\}$. Сопоставляя (3.21) с (3.23), замечаем, что

$$
p_{r-1, N}^{\alpha, \beta}(d, x)=p_{r-1, N}^{\beta, \alpha}(\bar{d}, N-1+2 r-x), \quad x \in\{r, r+1, \ldots, N-1+2 r\} .
$$

Поскольку $p_{r-1, N}^{\alpha, \beta}(d, x)$ и $p_{r-1, N}^{\beta, \alpha}(\bar{d}, N-1+2 r-x)$ представляют собой алгебраические полиномы степени $r-1<N$, из (3.24) следует, что они тождественно равны. Поэтому равенство (3.23) в сочетании с (3.21) дает

$$
d(x-r)=p_{r-1, N}^{\alpha, \beta}(d, x)+\mathscr{F}_{r, N}^{\alpha, \beta}(d, x), \quad x \in \Omega_{N+2 r}
$$

Тем самым, доказано, что равенство (3.14) справедливо для всех $x \in \Omega_{N+2 r}$. Подводя итоги, приходим к следуюшему результату. 
Tеорема 3.1. Пусть $\alpha, \beta>-1, \lambda=\alpha+\beta$ не иелое, $r \leqslant N, x \in \Omega_{N+2 r}$. Тогда для произвольной дискретной функиии $d=d(x)$, заданной на множестве $\bar{\Omega}_{N+2 r}=\{-r,-r+1, \ldots,-1,0,1, \ldots, N-1, N, \ldots, N-1+r\}$, имеет место равенство

$$
d(x-r)=Q_{r-1, N}(d, x)+A_{r-1, N}^{\alpha, \beta}(d, x)+\mathscr{F}_{r, N}^{\alpha, \beta}(d, x),
$$

где функиии $Q_{r-1, N}(d, x), A_{r-1, N}^{\alpha, \beta}(d, x), \mathscr{F}_{r, N}^{\alpha, \beta}(d, x)$ определены равенствами $(3.15)-$ (3.17).

Перейдем теперь к случаю, когда $\lambda=\alpha+\beta \in\{-1,0,1\}$. Перепишем (3.7) следуюшим образом:

$$
d(x-r)=Q_{r-1, N}(d, x)+B_{2 r-1-\lambda, N}^{\alpha, \beta}(d, x)+G_{r, N}^{\alpha, \beta}(d, x),
$$

где

$$
B_{2 r-1-\lambda, N}^{\alpha, \beta}(d, x)=\sum_{k=0}^{r-\lambda-1} \frac{d_{r, k}^{\alpha, \beta}}{\left\{h_{k, N+r}^{\alpha, \beta}\right\}^{1 / 2}} \frac{1}{(r-1) !} \sum_{t=0}^{x-1}(x-t)^{[r-1]} T_{k}^{\alpha, \beta}(t, N+r)
$$

- алгебраический полином степени $2 r-1-\lambda$,

$$
G_{r, N}^{\alpha, \beta}(d, x)=\sum_{k=r-\lambda}^{N-1+r} \frac{d_{r, k}^{\alpha, \beta}}{\left\{h_{k, N+r}^{\alpha, \beta}\right\}^{1 / 2}} \frac{1}{(r-1) !} \sum_{t=0}^{x-1}(x-t)^{[r-1]} T_{k}^{\alpha, \beta}(t, N+r) .
$$

Если $k+\lambda \geqslant r$, то $(k+\lambda)^{[r]}>0$ и для таких $k$ можем воспользоваться равенством $(3.8)$ и вытекаюшим отсюда равенством (3.13). Тогда (3.27) мы можем переписать так:

$$
G_{r, N}^{\alpha, \beta}(d, x)=E_{r-1, N}^{\alpha, \beta}(d, x)+\mathscr{F}_{r, N}^{\alpha, \beta}(d, x),
$$

где

$$
\begin{aligned}
E_{r-1, N}^{\alpha, \beta}(d, x)= & -\sum_{\nu=0}^{r-1}\left(\frac{(-1)^{r-\nu}(N+r)_{r-\nu}}{\nu ! \Gamma(\nu-r+\beta+1)}\right. \\
& \left.\times \sum_{k=r-\lambda}^{N-1+r} \frac{(-1)^{k} d_{r, k}^{\alpha, \beta}}{\left\{h_{k, N+r}^{\alpha, \beta}\right\}^{1 / 2}} \frac{\Gamma(k+\beta+1)(k+\lambda-r+1)_{\nu}}{(k+r-\nu) !(k+\lambda)^{[r]}}\right) x^{[\nu]} \\
\mathscr{F}_{r, N}^{\alpha, \beta}(d, x)= & (N-1+2 r)^{[r]} \sum_{k=r-\lambda}^{N-1+r} \frac{d_{r, k}^{\alpha, \beta}}{\left\{h_{k, N+r}^{\alpha, \beta}\right\}^{1 / 2}} \frac{T_{k+r}^{\alpha-r, \beta-r}(x, N+2 r)}{(k+\lambda)^{[r]}} .
\end{aligned}
$$

Сопоставляя (3.25)-(3.30), получаем следуюший смешанньй ряд в случае $\lambda=\alpha+\beta \in$ $\{-1,0,1\}$ :

$$
d(x-r)=Q_{r-1, N}(d, x)+B_{2 r-1-\lambda, N}^{\alpha, \beta}(d, x)+E_{r-1, N}^{\alpha, \beta}(d, x)+\mathscr{F}_{r, N}^{\alpha, \beta}(d, x),
$$

где $x \in \Omega_{N+r}$.

Если $2 r \leqslant N-1$, то совершенно аналогично тому, как это было сделано вьшше в случае дробного $\lambda$, доказьвается, что равенство (3.31) также верно для всех $x \in \Omega_{N+2 r}$. 
4. Смешанный ряд по полиномам $T_{n}^{0,0}(x, M)$. В случае $\alpha=\beta=0$ смешанный ряд (3.17) принимает следуюший вид $\left(\lambda=0, d_{r, k}=d_{r, k}^{0,0}\right)$ :

$$
\mathscr{F}_{r, N}(d, x)=\mathscr{F}_{r, N}^{0,0}(d, x)=(N-1+2 r)^{[r]} \sum_{k=r}^{N-1+r} \frac{d_{r, k}}{\left\{h_{k, N+r}^{0,0}\right\}^{1 / 2}} \frac{T_{k+r}^{-r,-r}(x, N+2 r)}{k^{[r]}} .
$$

С другой стороны, из (1.17) имеем

$$
T_{n}^{-r,-r}(x, N+2 r)=\frac{(-1)^{r} x^{[r]}(N+2 r-x-1)^{[r]}}{(N-1+2 r)^{[r]}(N-1+r)^{[r]}} T_{n-r}^{r, r}(x-r, N) .
$$

Из (4.1) и (4.2) находим

$$
\mathscr{F}_{r, N}(d, x)=\frac{(-1)^{r} x^{[r]}(N+2 r-x-1)^{[r]}}{(N-1+r)^{[r]}} \sum_{k=r}^{N-1+r} \frac{d_{r, k}}{k^{[r]}} \frac{T_{n-r}^{r, r}(x-r, N)}{\left\{h_{k, N+r}^{0,0}\right\}^{1 / 2}} .
$$

Подставляя это значение в (3.31), получаем

$$
d(x)=q_{2 r-1, N}(x)+\frac{(-1)^{r}(x+r)^{[r]}(N-1+r-x)^{[r]}}{(N-1+r)^{[r]}} \sum_{k=r}^{N-1+r} \frac{d_{r, k}}{k^{[r]}} \frac{T_{n-r}^{r, r}(x, N)}{\left\{h_{k, N+r}^{0,0}\right\}^{1 / 2}}
$$

где $x \in \bar{\Omega}_{N+2 r}$

$$
q_{2 r-1, N}(x)=Q_{r-1, N}(d, x+r)+B_{2 r-1, N}^{0,0}(d, x+r)+E_{r-1, N}^{0,0}(d, x+r)
$$

- полином степени $2 r-1$. Из (4.4) следует, что полином $q_{2 r-1, N}(x)$ совпадает с функцией $d(x)$ при $x \in\{-r, \ldots,-1\} \cup\{N, \ldots, N-1+r\}$. Поэтому $q_{2 r-1, N}(x)$ имеет вид

$$
q_{2 r-1, N}(x)=\sum_{i=1}^{r}(-1)^{i-1} \frac{(x+1)_{r}(N-x)_{r}}{(i-1) !(r-i) !(N+i)_{r}}\left[\frac{d(-i)}{x+i}+\frac{d(N-1+i)}{N-1+i-x}\right] .
$$

Сопоставляя (4.4) и (4.5), можем записать смешанньй ряд в следующем виде:

$$
\begin{aligned}
d(x)= & \sum_{i=1}^{r}(-1)^{i-1} \frac{(x+1)_{r}(N-x)_{r}}{(i-1) !(r-i) !(N+i)_{r}}\left[\frac{d(-i)}{x+i}+\frac{d(N-1+i)}{N-1+i-x}\right] \\
& +\frac{(-1)^{r}(x+1)_{r}(N-x)_{r}}{(N-1+r)^{[r]}} \sum_{k=r}^{N-1+r} \frac{d_{r, k}}{k^{[r]}} \frac{T_{n-r}^{r, r}(x, N)}{\left\{h_{k, N+r}^{0,0}\right\}^{1 / 2}}
\end{aligned}
$$

где $x \in \bar{\Omega}_{N+2 r}=\{-r, \ldots,-1,0,1, \ldots, N-1, N, \ldots, N-1+r\}$. 
5. Операторы $\mathscr{Y}_{n+2 r, N}^{\alpha, \beta}(d)$. Запишем равенства (3.14) и (3.24) в следуюшем виде:

$$
d(x)=q^{\alpha, \beta}(x)+\mathscr{F}_{r, N}^{\alpha, \beta}(d, x+r),
$$

где $q^{\alpha, \beta}(x)$ - некоторьй (явно заданньй) алгебраический полином степени не вьше, чем $2 r$, причем

$$
\operatorname{deg} q^{\alpha, \beta}(f, x)= \begin{cases}2 r-1-\lambda, & \lambda \in\{-1,0,1\} \\ r-1, & \lambda \text { дробное }\end{cases}
$$

$\lambda=\alpha+\beta$. Представим $\mathscr{F}_{r, N}^{\alpha, \beta}(d, x+r)$ в следующем виде:

$$
\mathscr{F}_{r, N}^{\alpha, \beta}(d, x+r)=\mathscr{F}_{r, N, n}^{\alpha, \beta}(d, x+r)+R_{r, N, n}^{\alpha, \beta}(d, x+r)
$$

где

$$
\begin{gathered}
\mathscr{F}_{r, N, n}^{\alpha, \beta}(d, x)=(N-1+2 r)^{[r]} \sum_{k=\bar{r}}^{n+r} \frac{d_{r, k}^{\alpha, \beta}}{\left\{h_{k, N+r}^{\alpha, \beta}\right\}^{1 / 2}} \frac{T_{k+r}^{\alpha-r, \beta-r}(x, N+2 r)}{(k+\lambda)^{[r]}}, \\
\bar{r}= \begin{cases}r-\lambda, \quad \lambda \in\{-1,0,1\} \\
0, \quad \lambda \text { дробное },\end{cases} \\
R_{r, N, n}^{\alpha, \beta}(d, x)=\mathscr{F}_{r, N}^{\alpha, \beta}(d, x)-\mathscr{F}_{r, N, n}^{\alpha, \beta}(d, x) .
\end{gathered}
$$

Через $\mathscr{Y}_{n+2 r, N}^{\alpha, \beta}(d, x)$ обозначим полином степени $n+2 r$, определенньй с помощью следующего равенства:

$$
\mathscr{Y}_{n+2 r, N}^{\alpha, \beta}(d)=\mathscr{Y}_{n+2 r, N}^{\alpha, \beta}(d, x)=q^{\alpha, \beta}(x)+\mathscr{F}_{r, N, n}^{\alpha, \beta}(d, x+r)
$$

где

$$
q^{\alpha, \beta}(x-r)= \begin{cases}Q_{r-1, N}(d, x)+A_{r-1, N}^{\alpha, \beta}(d, x), & \lambda \text { дробное }, \\ Q_{r-1, N}(d, x)+B_{2 r-1-\lambda, N}^{\alpha, \beta}(d, x)+E_{r-1, N}^{\alpha, \beta}(d, x), & \lambda \in\{-1,0,1\},\end{cases}
$$

а полиномы $Q_{r-1, N}(d, x), A_{r-1, N}^{\alpha, \beta}(d, x), B_{2 r-1-\lambda, N}^{\alpha, \beta}(d, x)$ и $E_{r-1, N}^{\alpha, \beta}(d, x)$ определены равенствами $(3.15),(3.16),(3.26)$ и (3.29) и имеют степени $r-1, r-1,2 r-1-\lambda$ и $r-1$ соответственно. Из (5.1), (5.3)-(5.6) имеем

$$
d(x-r)=\mathscr{Y}_{n+2 r, N}^{\alpha, \beta}(d, x-r)+R_{r, N, n}^{\alpha, \beta}(d, x) .
$$

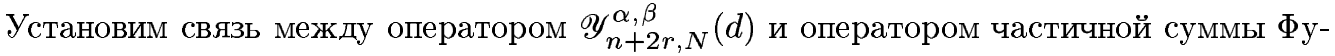
рье-Чебьшева $S_{n, N+2 r}^{\alpha, \beta}=S_{n, N+2 r}^{\alpha, \beta}(f)$, определенным в (2.5). Мы ограничимся здесь рассмотрением важного частного случая, когда $\alpha=\beta$. Перепишем сумму $\mathscr{F}_{r, N, n}^{\alpha}(d, x)=$ $\mathscr{F}_{r, N, n}^{\alpha, \alpha}(d, x)$, определенную равенством (5.4), следуюшим образом:

$$
\mathscr{F}_{r, N, n}^{\alpha}(d, x)=\mathscr{F}_{r, N, n-2 r}^{\alpha}(d, x)+I_{r, N, n}^{\alpha}(d, x),
$$


где $n \geqslant 2 r$,

$$
I_{r, N, n}^{\alpha}(d, x)=(N-1+2 r)^{[r]} \sum_{k=n-r+1}^{n+r} \frac{d_{r, k}^{\alpha, \alpha}}{\left\{h_{k, N+r}^{\alpha, \alpha}\right\}^{1 / 2}} \frac{T_{k+r}^{\alpha-r, \alpha-r}(x, N+2 r)}{(k+\lambda)^{[r]}} .
$$

Пользуясь леммой 1.1, можем записать

$$
\begin{aligned}
I_{r, N, n}^{\alpha}(d, x)= & \sum_{k=n-r+1}^{n+r} \frac{(N-1+2 r)^{[r]}}{(k+\lambda)^{[r]}} \frac{d_{r, k}^{\alpha, \alpha}}{\left\{h_{k, N+r}^{\alpha, \alpha}\right\}^{1 / 2}} \sum_{j=0}^{r} \lambda_{j}^{\alpha} T_{k+r-2 j}^{\alpha, \alpha}(x, N+2 r) \\
= & \varphi_{r, N, n}^{\alpha}(x)+\psi_{r, N, n}^{\alpha}(x), \\
\varphi_{r, N, n}^{\alpha}(x)= & \sum_{k=n-r+1}^{n+r} \frac{(N-1+2 r)^{[r]}}{(k+\lambda)^{[r]}} \frac{d_{r, k}^{\alpha, \alpha}}{\left\{h_{k, N+r}^{\alpha, \alpha}\right\}^{1 / 2}} \\
& \times \sum_{j=[(k+r-n-1) / 2]+1} \lambda_{j}^{\alpha} T_{k+r-2 j}^{\alpha, \alpha}(x, N+2 r) \\
\psi_{r, N, n}^{\alpha}(x)= & \sum_{k=n-r+1}^{n+r} \frac{(N-1+2 r)^{[r]}}{(k+\lambda)^{[r]}} \frac{d_{r, k}^{\alpha, \alpha}}{\left\{h_{k, N+r}^{\alpha, \alpha}\right\}^{1 / 2}} \\
& \times \sum_{j=0}^{[(k+r-n-1) / 2]} \lambda_{j}^{\alpha} T_{k+r-2 j}^{\alpha, \alpha}(x, N+2 r)
\end{aligned}
$$

где $[a]$ - целая часть числа $a$. Сопоставляя (5.6)-(5.9), можем записать

$$
\mathscr{Y}_{n+2 r, N}^{\alpha, \alpha}(d, x)=q^{\alpha, \alpha}(x)+\mathscr{F}_{r, N, n-2 r}^{\alpha}(d, x+r)+\varphi_{r, N, n}^{\alpha}(d, x+r)+\psi_{r, N, n}^{\alpha}(d, x+r),
$$

а из (5.7) имеем

$$
d(x)=\mathscr{Y}_{n+2 r, N}^{\alpha, \alpha}(d, x)+R_{r, N, n}^{\alpha}(d, x+r), \quad x \in \bar{\Omega}_{N+2 r} .
$$

Сопоставляя $(5.12)$ и (5.13), находим

$$
\begin{aligned}
d(x)= & q^{\alpha, \alpha}(x)+\mathscr{F}_{r, N, n-2 r}^{\alpha}(d, x+r)+\varphi_{r, N, n}^{\alpha}(d, x+r) \\
& +\psi_{r, N, n}^{\alpha}(d, x+r)+R_{r, N, n}^{\alpha}(d, x+r), \quad x \in \bar{\Omega}_{N+2 r} .
\end{aligned}
$$

Покажем, что $\left(x \in \bar{\Omega}_{N+2 r}\right)$

$$
q^{\alpha, \alpha}(x)+\mathscr{F}_{r, N, n-2 r}^{\alpha}(d, x+r)+\varphi_{r, N, n}^{\alpha}(d, x+r)=S_{n, N+2 r}^{\alpha}(\bar{d}, x+r),
$$

где $S_{n, N+2 r}^{\alpha}(\bar{d}, x+r)$ - частичная сумма Фурье функции $\bar{d}(x)=d(x-r)$ порядка $n$ по полиномам Чебышева $T_{k}^{\alpha, \alpha}(x, N+2 r), k=0,1, \ldots, N+2 r-1$ (см. (2.5)). В самом деле, левая часть равенства (5.15) представляет собой алгебраический полином степени $n$, поэтому

$$
q^{\alpha, \alpha}(x)+\mathscr{F}_{r, N, n-2 r}^{\alpha}(d, x+r)+\varphi_{r, N, n}^{\alpha}(d, x+r)=\sum_{l=0}^{n} v_{l} T_{l}^{\alpha, \alpha}(x+r, N+2 r) .
$$


Далее, из (5.11) следует, что

$$
\psi_{r, N, n}^{\alpha}(d, x+r)=\sum_{l=n+1}^{n+2 r} q_{l} T_{l}^{\alpha, \alpha}(x+r, N+2 r)
$$

а из (5.5) заключаем

$$
\begin{aligned}
R_{r, N, n}^{\alpha}(d, x+r) & =(N-1+2 r)^{[r]} \sum_{k=n+r+1}^{N-1+r} \frac{d_{r, k}^{\alpha, \alpha}}{\left\{h_{k, N+r}^{\alpha, \alpha}\right\}^{1 / 2}} \frac{T_{k+r}^{\alpha-r, \alpha-r}(x+r, N+2 r)}{(k+\lambda)^{[r]}} \\
& =\sum_{k=n+r+1}^{N-1+r} \frac{(N-1+2 r)^{[r]}}{(k+\lambda)^{[r]}} \frac{d_{r, k}^{\alpha, \alpha}}{\left\{h_{k, N+r}^{\alpha, \alpha}\right\}^{1 / 2}} \sum_{j=0}^{r} \lambda_{j}^{\alpha} T_{k+r-2 j}^{\alpha, \alpha}(x+r, N+2 r) \\
& =\sum_{l=n+1}^{N-1+2 r} a_{l} T_{l}^{\alpha, \alpha}(x+r, N+2 r) .
\end{aligned}
$$

Сопоставляя $(5.14),(5.16)-(5.18)$, замечаем, что

$$
\begin{aligned}
d(x)= & \sum_{l=0}^{n} v_{l} T_{l}^{\alpha, \alpha}(x+r, N+2 r)+\sum_{l=n+1}^{n+2 r}\left(q_{l}+a_{l}\right) T_{l}^{\alpha, \alpha}(x+r, N+2 r) \\
& +\sum_{l=n+2 r+1}^{N-1+2 r} a_{l} T_{l}^{\alpha, \alpha}(x+r, N+2 r),
\end{aligned}
$$

где $x \in \bar{\Omega}_{N+2 r}$. Заменим здесь $x$ на $x-r$; тогда

$$
\begin{aligned}
d(x-r)= & \sum_{l=0}^{n} v_{l} T_{l}^{\alpha, \alpha}(x, N+2 r)+\sum_{l=n+1}^{n+2 r}\left(q_{l}+a_{l}\right) T_{l}^{\alpha, \alpha}(x, N+2 r) \\
& +\sum_{l=n+2 r+1}^{N-1+2 r} a_{l} T_{l}^{\alpha, \alpha}(x, N+2 r), \quad x \in \Omega_{N+2 r} .
\end{aligned}
$$

Но, так как система $\left\{T_{l}^{\alpha, \alpha}(x, N+2 r)\right\}_{l=0}^{N-1+2 r}$ при $\alpha>-1$ ортогональна на $\Omega_{N+2 r}$, правая часть равенства (5.19) представляет собой разложение функции $\bar{d}(x)=d(x-r)$ в ряд Фурье по этой системе и, стало быть,

$$
\sum_{l=0}^{n} v_{l} T_{l}^{\alpha, \alpha}(x, N+2 r)=S_{n, N+2 r}^{\alpha}(\bar{d}, x), \quad x \in \Omega_{N+2 r}
$$

Сравнивая (5.16) с (5.20), приходим к равенству (5.15). Тогда (5.14) мы можем переписать так:

$$
d(x)=S_{n, N+2 r}^{\alpha}(\bar{d}, x+r)+\psi_{r, N, n}^{\alpha}(d, x+r)+R_{r, N, n}^{\alpha}(d, x+r), \quad x \in \bar{\Omega}_{N+2 r}
$$


Отсюда и из (5.13) выводим искомую связь между оператором $\mathscr{Y}_{n+2 r, N}^{\alpha}(d)=\mathscr{Y}_{n+2 r, N}^{\alpha, \alpha}(d)$ и оператором частичной суммы $\Phi$ урье $S_{n, N+2 r}^{\alpha}(\bar{d})$ :

$$
\mathscr{Y}_{n+2 r, N}^{\alpha}(d, x)=S_{n, N+2 r}^{\alpha}(\bar{d}, x+r)+\psi_{r, N, n}^{\alpha}(d, x+r) .
$$

Другое важное свойство операторов $\mathscr{Y}_{n+2 r, N}^{\alpha, \beta}(d)=\mathscr{Y}_{n+2 r, N}^{\alpha, \beta}(d, x)$ выражается равенством

$$
\begin{aligned}
& \Delta^{\nu} d(x-r)-\Delta^{\nu} \mathscr{Y}_{n+2 r, N}^{\alpha, \beta}(d, x-r) \\
& \quad=R_{r-\nu, N+\nu, n+\nu}^{\alpha, \beta}\left(\Delta^{\nu} d, x\right) \\
& \quad=\sum_{k=n+r+1}^{N-1+r} \frac{(N+\nu-1+2(r-\nu))^{[r-\nu]} d_{r, k}^{\alpha, \beta}}{(k+\lambda)^{[r-\nu]}\left\{h_{k, N+r}^{\alpha, \beta}\right\}^{1 / 2}} T_{k+r-\nu}^{\alpha-r+\nu, \beta-r+\nu}(x, N+\nu+2(r-\nu)),
\end{aligned}
$$

где $0 \leqslant \nu \leqslant r-1$. В самом деле, из (5.7) имеем

$$
\begin{aligned}
d(x-r)-\mathscr{Y}_{n+2 r, N}^{\alpha, \beta}(d, x-r)=R_{r, N, n}^{\alpha, \beta}(d, x) \\
\quad=\sum_{k=n+r+1}^{N-1+r} \frac{(N-1+2 r)^{[r]} d_{r, k}^{\alpha, \beta}}{(k+\lambda)^{[r]}\left\{h_{k, N+r}^{\alpha, \beta}\right\}^{1 / 2}} T_{k+r}^{\alpha-r, \beta-r}(x, N+2 r)
\end{aligned}
$$

и, стало быть,

$$
\begin{aligned}
& \Delta^{\nu} d(x-r)-\Delta^{\nu} \mathscr{Y}_{n+2 r, N}^{\alpha, \beta}(d, x-r) \\
& \quad=\sum_{k=n+r+1}^{N-1+r} \frac{(N-1+2 r)^{[r]} d_{r, k}^{\alpha, \beta}}{(k+\lambda)^{[r]}\left\{h_{k, N+r}^{\alpha, \beta}\right\}^{1 / 2}} \Delta^{\nu} T_{k+r}^{\alpha-r, \beta-r}(x, N+2 r) .
\end{aligned}
$$

Если мы воспользуемся равенством (1.16), то получим отсюда (5.23).

6. Аппроксимативные свойства операторов $\mathscr{Y}_{n+2 r, N}^{\alpha, \beta}$. Будем рассматривать оператор $\mathscr{Y}_{n+2 r, N}^{\alpha, \beta}=\mathscr{Y}_{n+2 r, N}^{\alpha, \beta}(d)$ как апшарат приближения дискретных функций, заданных на сетке $\bar{\Omega}_{N+2 r}$. При этом нам понадобятся некоторые обозначения. Положим

$$
\begin{aligned}
B_{n, r, \nu}^{\alpha, \beta}(x, N) & =\max \left\{\frac{\left|T_{k+r-\nu}^{\alpha-r+\nu, \beta-r+\nu}(x, N+2 r-\nu)\right|}{\sqrt{h_{k, N+r}^{\alpha, \beta}}}: n+r+1 \leqslant k \leqslant N-1+r\right\} \\
H_{n, r, \nu}^{\alpha, \beta}(x, N) & =\frac{\left(\sqrt{\frac{x+1}{N}}+\frac{1}{n}\right)^{\beta}\left(\sqrt{1-\frac{x}{N+2 r}}+\frac{1}{n}\right)^{\alpha}}{\left(\sqrt{\frac{x+1}{N}}+\frac{1}{n}\right)^{r-\nu-1 / 2}\left(\sqrt{1-\frac{x}{N+2 r}}+\frac{1}{n}\right)^{r-\nu-1 / 2}} B_{n, r, \nu}^{\alpha, \beta}(x, N), \\
\chi_{n, r, \nu}^{\alpha, \beta}(N) & =\max \left\{H_{n, r, \nu}^{\alpha, \beta}(x, N): x \in \Omega_{N+2 r}\right\} .
\end{aligned}
$$

Из (6.2) и (6.3) следует, что

$B_{n, r, \nu}^{\alpha, \beta}(x, N) \leqslant \chi_{n, r, \nu}^{\alpha, \beta}(N)\left(\sqrt{\frac{x+1}{N}}+\frac{1}{n}\right)^{-\beta+r-\nu-1 / 2}\left(\sqrt{1-\frac{x}{N+2 r}}+\frac{1}{n}\right)^{-\alpha+r-\nu-1 / 2}$. 
С произвольной дискретной функцией $d=d(x)$, заданной на сетке $\bar{\Omega}_{N+2 r}$, мы свяжем дискретную функцию

$$
g(x)=\frac{N-1+2 r}{2} \Delta^{r} d(x-r),
$$

определенную на $\Omega_{N+r}$. Пусть $E_{n+r}(g)_{\ell_{2, \mu}^{N+r}}$ представляет собой наилучшее приближение функции $g(x)$ в пространстве $\ell_{2, \mu}^{N+r}$ алгебраическими полиномами степени $n+r$. Тогда мы можем сформулировать следуюший результат.

Теорема 6.1. Если $\alpha, \beta>-1, \lambda=\alpha+\beta, 1 \leqslant r$ иелое, $0 \leqslant \nu \leqslant r-1, x \in \Omega_{N+2 r-\nu}$, то имеет место оценка

$$
\begin{aligned}
& \left(\frac{N-1+2 r}{2}\right)^{\nu}\left|\Delta^{\nu} d(x-r)-\Delta^{\nu} \mathscr{Y}_{n+2 r, N}^{\alpha, \beta}(d, x-r)\right| \\
& \quad \leqslant B_{n, r, \nu}^{\alpha, \beta}(x, N) 2^{r-\nu}\left(\sum_{k=n+r+1}^{N-1+r} \frac{1}{\left((k+\lambda)^{[r-\nu]}\right)^{2}}\right)^{1 / 2} E_{n+r}(g)_{\ell_{2, \mu}^{N+r}} .
\end{aligned}
$$

ДокАЗАтЕльство. Обратимся к равенству (5.23). С учетом введенных вьше обозначений имеем

$$
\left|\Delta^{\nu} d(x-r)-\Delta^{\nu} \mathscr{Y}_{n+2 r, N}^{\alpha, \beta}(d, x-r)\right| \leqslant B_{n, r, \nu}^{\alpha, \beta}(x, N) \sum_{k=n+r+1}^{N-1+r} \frac{(N-1+2 r-\nu)^{[r-\nu]}}{(k+\lambda)^{[r-\nu]}}\left|d_{r, k}^{\alpha, \beta}\right| .
$$

Отсюда имеем $(0 \leqslant \nu \leqslant r-1)$

$$
\begin{aligned}
& \left(\frac{N-1+2 r}{2}\right)^{\nu}\left|\Delta^{\nu} d(x-r)-\Delta^{\nu} \mathscr{Y}_{n+2 r, N}^{\alpha, \beta}(d, x-r)\right| \\
& \leqslant B_{n, r, \nu}^{\alpha, \beta}(x, N)\left(\frac{N-1+2 r}{2}\right)^{\nu} \sum_{k=n+r+1}^{N-1+r} \frac{(N-1+2 r-\nu)^{[r-\nu]}\left|d_{r, k}^{\alpha, \beta}\right|}{(k+\lambda)^{[r-\nu]}} \\
& \leqslant B_{n, r, \nu}^{\alpha, \beta}(x, N)\left(\frac{N-1+2 r}{2}\right)^{r} \sum_{k=n+r+1}^{N-1+r} \frac{2^{r-\nu}\left|d_{r, k}^{\alpha, \beta}\right|}{(k+\lambda)^{[r-\nu]}} \\
& \leqslant B_{n, r, \nu}^{\alpha, \beta}(x, N) 2^{r-\nu}\left(\sum_{k=n+r+1}^{N-1+r} \frac{1}{\left((k+\lambda)^{[r-\nu]}\right)^{2}}\right)^{1 / 2} \\
& \quad \times\left(\sum_{k=n+r+1}^{N-1+r}\left[\left(\frac{N-1+2 r}{2}\right)^{r} d_{r, k}^{\alpha, \beta}\right]^{2}\right)^{1 / 2} \cdot
\end{aligned}
$$

В силу (3.2)-(3.4) замечаем, что

$$
\left(\sum_{k=n+r+1}^{N-1+r}\left[\left(\frac{N-1+2 r}{2}\right)^{r} d_{r, k}^{\alpha, \beta}\right]^{2}\right)^{1 / 2}=E_{n+r}(g)_{\ell_{2, \mu}^{N+r}}
$$

Сопоставляя (6.6) и (6.7), приходим к утверждению теоремы 6.1. 
СЛЕДСТВИЕ 6.1. В условиях теоремы 6.1 имеет место следующая оценка $(x \in$ $\Omega_{N+2 r-\nu)}$

$$
\begin{aligned}
& \left(\frac{N-1+2 r}{2}\right)^{\nu}\left|\Delta^{\nu} d(x-r)-\Delta^{\nu} \mathscr{Y}_{n+2 r, N}^{\alpha, \beta}(d, x-r)\right| \\
& \leqslant \\
& \quad c(\lambda, r) \chi_{n, r, \nu}^{\alpha, \beta}(N) \frac{E_{n+r}(g)_{\ell_{2, \mu}^{N+r}}}{n^{r-\nu-1 / 2}}\left(\sqrt{\frac{x+1}{N}}+\frac{1}{n}\right)^{-\beta+r-\nu-1 / 2} \\
& \quad \times\left(\sqrt{1-\frac{x}{N+2 r}}+\frac{1}{n}\right)^{-\alpha+r-\nu-1 / 2}
\end{aligned}
$$

где $c(\lambda, r)$ - полохстельное число, зависящее лишь от указанных параметров.

В самом деле, из теоремы 6.1 нетрудно увидеть, что

$$
\left(\frac{N-1+2 r}{2}\right)^{\nu}\left|\Delta^{\nu} d(x-r)-\Delta^{\nu} \mathscr{Y}_{n+2 r, N}^{\alpha, \beta}(d, x-r)\right| \leqslant c(\lambda, r) B_{n, r, \nu}^{\alpha, \beta}(x, N) \frac{E_{n+r}(g)_{\ell_{2, \mu}^{N+r}}}{n^{r-\nu-1 / 2}} .
$$

Если мы воспользуемся оценкой (6.4), то из (6.9) получим (6.8).

ЗАмЕчаниЕ. Эксперимент, проведенный с помощью ЭВМ, показал, что число $\chi_{n, r, \nu}^{\alpha, \beta}(N)$ не имеет тенденции к неограниченному росту при $N, n \rightarrow \infty$.

7. Операторы $\mathscr{X}_{n+2 r, N}^{\alpha, \beta}(f)$. В предыдуших пунктах были рассмотрены операторы $\mathscr{Y}_{n+2 r, N}^{\alpha, \beta}(d)$, действуюшие в пространствах дискретных функций вида $d: \bar{\Omega}_{N+2 r} \rightarrow \mathbb{R}$. Отправляясь от этих операторов, построим здесь операторы $\mathscr{X}_{n+2 r, N}^{\alpha, \beta}(f)$, действующие в пространстве непрерьвных функций $C[-1,1]$, и исследуем их аппроксимативные свойства. Пусть функция $f=f(x)$ определена в узлах сетки

$$
H_{\Lambda}=\left\{x_{j}=-1+\frac{2 j}{\Lambda-1}\right\}_{j=0}^{\Lambda-1}
$$

где $\Lambda=N+2 r, N, r \in \mathbb{N}$. Определим на сетке $\bar{\Omega}_{\Lambda}=\{-r,-r+1, \ldots,-1,0,1, \ldots, N, \ldots$, $N-1+r\}$ дискретную функцию $d: \bar{\Omega}_{\Lambda} \rightarrow \mathbb{R}$ следуюшим образом:

$$
d(j-r)=f\left(x_{j}\right), \quad j \in \Omega_{\Lambda} .
$$

Тогда для функции $d=d(j)$ мы можем построить полином $\mathscr{Y}_{n+2 r, N}^{\alpha, \beta}(d)$ с помошью равенства (5.6). Положим

$$
\mathscr{X}_{n+2 r, N}^{\alpha, \beta}(f)=\mathscr{X}_{n+2 r, N}^{\alpha, \beta}(f, x)=\mathscr{Y}_{n+2 r, N}^{\alpha, \beta}\left(d, \frac{\Lambda-1}{2}(1+x)-r\right) .
$$

Ясно, что $\mathscr{X}_{n+2 r, N}^{\alpha, \beta}(f, x)$ вместе с $\mathscr{Y}_{n+2 r, N}^{\alpha, \beta}(d, t)$ представляет собой алгебраический полином степени $n+2 r$. Поэтому мы можем рассмотреть $\mathscr{X}_{n+2 r, N}^{\alpha, \beta}(f)$ как оператор, действуюший в пространстве $C[-1,1]$. Отметим следуюшие свойства оператора $\mathscr{X}_{n+2 r, N}^{\alpha, \beta}(f)$, непосредственно вытекаюшие из свойств оператора $\mathscr{Y}_{n+2 r, N}^{\alpha, \beta}(d)$. Из (7.2) и (5.7) имеем

$$
\begin{aligned}
f\left(x_{j}\right)-\mathscr{X}_{n+2 r, N}^{\alpha, \beta}\left(f, x_{j}\right) & =d(j-r)-\mathscr{Y}_{n+2 r, N}^{\alpha, \beta}(d, j-r)=R_{r, N, n}^{\alpha, \beta}(d, j) \\
& =(N-1+2 r)^{[r]} \sum_{k=n+r+1}^{N-1+r} \frac{d_{r, k}^{\alpha, \beta}}{\left\{h_{k, N+r}^{\alpha, \beta}\right\}^{1 / 2}} \frac{T_{k+r}^{\alpha-r, \beta-r}(j, N+2 r)}{(k+\lambda)^{[r]}} .
\end{aligned}
$$


Если мы положим $\sigma=2 /(N-1+2 r)$, то из (5.23) в сочетании с (7.1) выводим

$$
\begin{aligned}
& \Delta_{\sigma}^{\nu} f\left(x_{j}\right)-\Delta_{\sigma}^{\nu} \mathscr{X}_{n+2 r, N}^{\alpha, \beta}\left(f, x_{j}\right) \\
& \quad=\Delta^{\nu} d(j-r)-\Delta^{\nu} \mathscr{Y}_{n+2 r, N}^{\alpha, \beta}(d, j-r) \\
& \quad=\sum_{k=n+r+1}^{N-1+r} \frac{(N+\nu-1+2(r-\nu))^{[r-\nu]} d_{r, k}^{\alpha, \beta}}{(k+\lambda)^{[r-\nu]}\left\{h_{k, N+r}^{\alpha, \beta}\right\}^{1 / 2}} T_{k+r-\nu}^{\alpha-r+\nu, \beta-r+\nu}(j, N+\nu+2(r-\nu))
\end{aligned}
$$

где $0 \leqslant \nu \leqslant r-1, \Delta_{\sigma}^{\nu}-$ конечная разность порядка $\nu$ с шагом $\sigma$. Если мы обратимся к оценке (6.8), то из (7.4) получим $(0 \leqslant j \leqslant N+2 r-\nu-1)$

$$
\begin{aligned}
\sigma^{-\nu} \mid & \Delta_{\sigma}^{\nu} f\left(x_{j}\right)-\Delta_{\sigma}^{\nu} \mathscr{X}_{n+2 r, N}^{\alpha, \beta}\left(f, x_{j}\right) \mid \\
\leqslant & c(\lambda, r) \chi_{n, r, \nu}^{\alpha, \beta}(N) \frac{E_{n+r}(g)_{\ell_{2, \mu}^{N+r}}}{n^{r-\nu-1 / 2}}\left(\sqrt{\frac{j+1}{N}}+\frac{1}{n}\right)^{-\beta+r-\nu-1 / 2} \\
& \times\left(\sqrt{1-\frac{j}{N+2 r}}+\frac{1}{n}\right)^{-\alpha+r-\nu-1 / 2}
\end{aligned}
$$

где $g(j)=\sigma^{-\nu} \Delta^{r} d(j-r)$. Отсюда, воспользовавшись известной формулой

$$
\sigma^{-\nu}\left[\Delta_{\sigma}^{\nu} f\left(x_{j}\right)-\Delta_{\sigma}^{\nu} \mathscr{X}_{n+2 r, N}^{\alpha, \beta}\left(f, x_{j}\right)\right]=f^{(\nu)}\left(x_{j}+\nu \sigma \theta_{j}\right)-\left(\mathscr{X}_{n+2 r, N}^{\alpha, \beta}\left(f, x_{j}+\nu \sigma \theta_{j}\right)\right)^{(\nu)}
$$

где $0<\theta_{j}<1$, в предположении $\nu$-кратной непрерьвной дифференцируемости функции $f=f(x)$ получим

$$
\begin{aligned}
& \left|f^{(\nu)}\left(x_{j}+\nu \sigma \theta_{j}\right)-\left(\mathscr{X}_{n+2 r, N}^{\alpha, \beta}\left(f, x_{j}+\nu \sigma \theta_{j}\right)\right)^{(\nu)}\right| \\
& \leqslant \\
& \quad c(\lambda, r) \chi_{n, r, \nu}^{\alpha, \beta}(N) \frac{E_{n+r}(g)_{\ell_{2, \mu}^{N+r}}}{n^{r-\nu-1 / 2}}\left(\sqrt{\frac{j+1}{N}}+\frac{1}{n}\right)^{-\beta+r-\nu-1 / 2} \\
& \quad \times\left(\sqrt{1-\frac{j}{N+2 r}}+\frac{1}{n}\right)^{-\alpha+r-\nu-1 / 2},
\end{aligned}
$$

где $0<\theta_{j}<1,0 \leqslant j \leqslant N+2 r-\nu$.

\section{СПИСОК ЦИТИРОВАННОЙ ЛИТЕРАТУРЫ}

[1] Шарапудинов И. И.Приближений функций с переменной гладкостью суммами Фурье по ортогональным полиномам // Международная конференция "Теория приближений и гармонический анализ" (26-29 мая 1998г.). Тула, 1998. С. 275.

[2] Шарапудинов И.И.Приближение дискретных функций и многочлены Чебьшева, ортогональные на равномерной сетке // Воронежская зимняя математическая школа "Современные методы теории функций и смежные проблемы”. Тезисы докладов ( 27 января-4 февраля 1999г.). Воронеж, 1999. С. 204.

[3] Шарапудинов И.И. Исправленные суммы Фурье по ортогональным полиномам и их аппроксимативные свойства // Воронежская зимняя математическая школа "Современные методы теории функций и смежные проблемы". Тезисы докладов (27 января-4 февраля 2001г.). Воронеж, 2001. С. 289-290. 
[4] Шарапудинов И.И. Смешанные ряды по ортогональным полиномам // Современные проблемы теории функций и их приложения. Тезисы докладов 10 -й Саратовской зимней школы (28 января-4 февраля 2002 г.). Саратов, 2002. С. 228-229.

[5] Шарапудинов И.И. Смешанные ряды по полиномам, ортогональньм на дискретных сетках // Воронежская зимняя математическая школа "Современные методы теории функций и смежные проблемы”. Тезисы докладов (26 января-2 февраля 2003г.). Воронеж, 2003. C. $285-286$.

[6] Шарапудинов И.И. Смешанные ряды по полиномам Якоби и методы их дискретизации в Чебышевском случае // Труды математического центра им. Н. И. Лобачевского. Т. 19. Теория функций, ее приложения и смежные вопросы. Материалы шестой Казанской международной летней школы-конференции (Казань, 27 июня-4 июля 2003г.). Казань: Изд-во Казанского матем. об-ва, 2003.

[7] Шарапудинов И. И. Смешанные ряды по некоторым ортогональньм системам и их приложения // Современные проблемы теории функций и их приложения. Тезисы докладов 12 -й Саратовской зимней школы (27 января-3 февраля 2004г.). Саратов: Изд-во ГосУНЦ "Колледж", 2004. С. 205-206.

[8] Шарапудинов И. И. Смешанные ряды по ульрасферическим полиномам и их аппроксимативные свойства // Матем. сб. 2003. Т. 194. № 3. С. 115-148.

[9] Шарапудинов И.И. Аппроксимативные свойства операторов $\mathscr{Y}_{n+2 r}(f)$ и их дискретных аналогов // Матем. заметки. 2002. Т. 72. № 5. С. 765-795.

[10] Шарапудинов И.И. Многочлены, ортогональные на дискретных сетках. Махачкала: Изд-во Даг. гос. пед. ун-та, 1997.

[11] Karlin S., McGregor J. L. The Hahn polynomials, formulas and an application // Scripta Math. 1961. V. 26. №1. P. 33-46.

[12] Gasper G. Positivity and special functions // Theory and Application of Special Functions Proc. Advanced Sem., Math. Res. Center, Univ. Wisconsin, Madison, Wis., 1975) / ed. R. A. Askey. Math. Res. Center, Univ. Wisconsin, Publ. No. 35. New York: Academic Press, 1975. P. 375-433.

[13] Сегё Г. Ортогональные многочлены. М.: Физматгиз, 1962.

Дагестанский государственный педагогический университет,

Поступило

г. Махачкала

29.04.2004 